\title{
ON THE CALCULATION OF STEADY-STATE LOSS PROBABILITIES IN THE GI/G/2/0 QUEUE
}

\author{
IGOR N. KOVALENKO \\ Ukrainian National Academy of Sciences \\ Institute of Cybernetics \\ 40 Prospekt Glushkova, 252007 Kiev, UKRAINE \\ J. BEN ATKINSON \\ University of North London \\ School of Mathematical Sciences \\ Holloway Road, London N7 8DB, UK
}

(Received March, 1994; revised June, 1994)

\begin{abstract}
This paper considers methods for calculating the steady-state loss probability in the GI/G/2/0 queue. A previous study analyzed this queue in discrete time and this led to an efficient, numerical approximation scheme for continuous-time systems. The primary aim of the present work is to provide an alternative approach by analyzing the GI/ME/2/0 queue; i.e., assuming that the service time can be represented by a matrix-exponential distribution. An efficient computational scheme based on this method is developed and some numerical examples are studied. Some comparisons are made with the discrete-time approach, and the two methods are seen to be complementary.
\end{abstract}

Key words: Matrix-exponential, Discrete-time Approximation, Loss Probability, GI/G/2/0 queue.

AMS (MOS) subject classifications: $60 \mathrm{~K} 25,90 \mathrm{~B} 22$.

\section{Introduction}

In this paper we consider methods for calculating the steady-state loss probability in the GI/G/2/0 queue. In a previous study of this system [3], numerically exact probabilities were obcained for discrete-time systems in which both the interarrival time and the service time take finite integer values, and this led to an effective numerical approximation scheme for continuoustime systems.

Our primary aim in the present work is to provide an alternative method for solving continuous-time problems. We do this by using a matrix-exponential representation of the service-time distribution; i.e., by studying the GI/ME/2/0 queue. A matrix exponential density function $f(x)$ has the form $f(x)=\underline{a} \exp (\underline{T} x) \underline{s}$, where $\underline{a}$ is a row vector, $\underline{s}$ is a column vector and $\underline{T}$ is a matrix, and complex entries are allowed in $a, s$ and $T$. An equivalent characterization is

\footnotetext{
${ }^{1}$ This author's research for this paper was supported by the British Council
} 
that $f(x)$ has a rational Laplace transform. The matrix exponential distribution is identical in form to the widely-used phase-type distribution (see Neuts [5]), except that in the latter case the entries in $a, s$ and $T$ are subject to additional restrictions. Both matrix-exponential and phasetype distributions are powerful models for approximating general probability density functions in queueing and related processes. In the next section, we give a detailed steady-state analysis of the GI/ME/2/0 queue, leading to two computational schemes for the calculation of loss probabilities. This is followed, for comparative purposes, by a brief description of the discrete-time model referred to above. Some preliminary, numerical studies are then presented to illustrate both the discrete-time and matrix-exponential approaches and to make some comparisons between them.

\section{The Queueing System GI/ME/2/0}

We begin with some notation:

$A(x)$ - distribution function of interarrival time,

$b(x) \quad$ - probability density function of service time,

$t_{n} \quad$ - arrival time of customer $n$,

$p_{\text {loss }}$ - steady-state loss probability; the desired value,

$\nu_{n} \quad$ - number of busy channels at time $t_{n}+0$,

$\xi_{n} \quad$ - service time of customer $n$ if $\nu_{n}=1$,

$\xi_{n}^{(1)}, \xi_{n}^{(2)}$ - residual service times in channels 1 and 2 respectively, if $\nu_{n}=2$; in such cases $\xi_{n}=\left(\xi_{n}^{(1)}, \xi_{n}^{(2)}\right)$ is a vector.

We make the following assumptions:

(1) Both the mean interarrival time $a$ and the mean service time $s$ are finite; moreover $a>0$. $b(x)$ takes the following matrix-exponential form:

$$
b(x)=\sum_{i=1}^{m} \exp \left(-\lambda_{i} x\right) \sum_{k=1}^{r_{i}} b_{i k} \lambda_{i}^{k} x^{k-1} /(k-1) !, \quad x>0
$$

where it suffices to assume that $\operatorname{Re}\left(\lambda_{i}\right)>0$ and the right-hand side of (1) should be nonnegative for all $x>0$.

Obviously the sequence $\left(\nu_{n}, \xi_{n}\right)$ is a homogeneous Markov chain. One could easily derive integral equations for its steady-state distribution, but we shall use Cox's principle of complex probabilities [4], following which we can write down steady-state equations as if $\lambda_{i}$ were positive and $b_{i k} \geq 0$ and summing up to 1 . Thus each customer arrival originates a trial with outcome probabilities $b_{i k}$, so that in the $(i, k)$ case the service time is the sum of $k$ independent exponential phases having parameter $\lambda_{i}$. Therefore, instead of the variables $\xi_{n}$, one may consider the embedded Markov chain $\left(\nu_{n}, i_{n}, j_{n}\right)$ in which $\nu_{n}$ has the same meaning as above. If $\nu_{n}=1$ then, for the customer in service at time $t_{n}+0$, the parameter of its exponential phase is $\lambda_{i_{n}}$ (i.e., having index $i_{n}$ ), while $j_{n}$ is the number of residual phases including the current one. If $\nu_{n}=2$, then $i_{n}=\left(i_{n}^{(1)}, i_{n}^{(2)}\right), j_{n}=\left(j_{n}^{(1)}, j_{n}^{(2)}\right)$ where $i_{n}^{(1)}$ and $i_{n}^{(2)}$ index the parameters of the exponential phases in channels 1 and 2 respectively, while $j_{n}^{(1)}$ and $j_{n}^{(2)}$ are the corresponding numbers of residual phases in channels 1 and 2 .

The steady-state equations can be obtained from the following transition diagram of the Markov chain: 


$$
\text { from }(1, i, j)\left\{\begin{array}{c}
\text { to }\left(1, i^{\prime}, j^{\prime}\right) \text { with prob. } f_{i, \geq j^{\prime} b_{i^{\prime} j^{\prime}}} \\
\text { to }\left(2,\left(i^{\prime}, i^{\prime \prime}\right),\left(j^{\prime}, j^{\prime \prime}\right)\right) \text { with prob. } \\
\frac{1}{2}\left(\delta_{i i^{\prime}} f_{i, j-j^{\prime}} b_{i^{\prime \prime} j^{\prime \prime}}+\delta_{i i^{\prime \prime}} f_{i, j-j^{\prime \prime}} b_{i^{\prime} j^{\prime}}\right)
\end{array}\right.
$$

$$
\begin{aligned}
& \text { to }\left(1, i^{\prime}, j^{\prime}\right) \text { with prob. } F_{i}{ }_{i\left(i^{(1)}\right.}{ }^{(2)}, \geq j^{(1)}, \geq j^{(2)^{b}} i^{\prime} j^{\prime} \\
& \text { to }\left(2,\left(i^{\prime}, i^{\prime \prime}\right),\left(j^{\prime}, j^{\prime \prime}\right)\right) \text { with prob. } \\
& \text { from }\left(2,\left(i^{(1)}, i^{(2)}\right),\left(j^{(1)}, j^{(2)}\right)\right) \\
& { }_{i}^{\delta}(1)_{i^{\prime}}{ }_{i}(2)_{i^{\prime \prime}}{ }^{F}{ }_{i}(1), i(2), j^{(1)}-j^{\prime}, j^{(2)}-j^{\prime \prime} \\
& +\delta_{i^{(1)} i_{i^{\prime}}}{ }_{i}{ }_{i}(1), i^{(2)}, j^{(1)}-j^{\prime}, \geq j^{(2)^{b}} i^{\prime} j^{\prime} \\
& +\delta_{i\left({ }_{i}\right)^{\prime \prime}} F_{i}(1), i(2), \geq j^{(1)}, j^{(2)}-j^{\prime \prime}{ }^{b} i^{\prime \prime} j^{\prime \prime}
\end{aligned}
$$

where $\delta_{i j}$ is the Kronecker delta,

$$
\begin{aligned}
& f_{i j}=\frac{1}{j !} \int_{0}^{\infty}\left(\lambda_{i} t\right)^{j} \exp \left(-\lambda_{i} t\right) d A(t) \\
& f_{i, \geq j}=1-\sum_{j^{\prime}=0}^{j-1} f_{i j^{\prime}} \\
& F_{i_{1}, i_{2}, j_{1}, j_{2}}=\frac{1}{j_{1} ! j_{2} !} \int_{0}^{\infty}\left(\lambda_{i_{1}} t\right)^{j_{1}}\left(\lambda_{i_{2}} t\right)^{j_{2}} \exp \left(-\lambda_{i_{1}} t-\lambda_{i_{2}} t\right) d A(t) \\
& F_{i_{1}, i_{2}, \geq j_{1}, j_{2}}=f_{i_{2} j_{2}}-\sum_{j^{\prime}=0}^{j_{1}-1} F_{i_{1}, i_{2}, j^{\prime}, j_{2}} \\
& F_{i_{1}, i_{2}, j_{1}, \geq j_{2}}=f_{i_{1} j_{1}}-\sum_{j^{\prime}=0}^{j_{2}-1} F_{i_{1}, i_{2}, j_{1}, j^{\prime}} \\
& F_{i_{1}, i_{2}, \geq j_{1} \geq j_{2}}=f_{i_{1}, \geq j_{1}}-\sum_{j^{\prime}=0}^{j_{2}-1} F_{i_{1}, i_{2}, \geq j_{1}, j^{\prime}}
\end{aligned}
$$

Thus, for example, $f_{i j}$ is the probability of completing $j$ exponential phases within an interarrival interval, the parameter of the exponential law being $\lambda_{i}$. The above transition diagram implies the following steady-state equations: 


$$
\begin{aligned}
& x(1, i, j)=\sum_{i^{\prime}, j^{\prime}} x\left(1, i^{\prime}, j^{\prime}\right) f_{i^{\prime}, \geq j^{\prime}} b_{i j} \\
& +\sum_{i^{\prime}, i^{\prime \prime}, j^{\prime}, j^{\prime \prime}} x\left(2, i^{\prime}, i^{\prime \prime}, j^{\prime}, j^{\prime \prime}\right) F_{i^{\prime}, i^{\prime \prime}, \geq j^{\prime}, \geq j^{\prime \prime}} b_{i j} \\
& x\left(2, i_{1}, i_{2}, j_{1}, j_{2}\right)=\sum_{k=0}^{r_{i_{1}}-j_{1}} \sum_{l=0}^{r_{i_{2}}-j_{2}} F_{i_{1} i_{2} k l} x\left(2, i_{1}, i_{2}, j_{1}+k, j_{2}+\ell\right) \\
& +\sum_{k=0}^{r_{i_{1}}-j_{1}} \sum_{i^{\prime}} \sum_{\ell=0}^{r_{i^{\prime}}} F_{i_{1}, i^{\prime}, k, \geq \ell^{b_{i_{2} j_{2}}}} x\left(2, i_{1}, i^{\prime}, j_{1}+k, l\right) \\
& +\sum_{k=0}^{r_{i_{2}}-j_{2}} \sum_{i^{\prime}} \sum_{\ell=0}^{r_{i^{\prime}}} F_{i^{\prime}, i_{2}, k, \geq \ell, k} b_{i_{1} j_{1}} x\left(2, i^{\prime}, i_{2}, l, j_{2}+k,\right) \\
& +\frac{1}{2} \sum_{k=0}^{r_{i_{1}}-j_{1}} f_{i_{1} k} b_{i_{2} j_{2}} x\left(1, i_{1}, j_{1}+k\right) \\
& +\frac{1}{2} \sum_{k=0}^{r_{i_{2}}-j_{2}} f_{i_{2} b_{i_{1} j_{1}}} x\left(1, i_{2}, j_{2}+k\right) \\
& \sum_{i_{1}, j_{1}} x\left(1, i_{1}, j_{1}\right)+\sum_{i_{1}, i_{2}, j_{1}, j_{2}} x\left(2, i_{1}, i_{2}, j_{1}, j_{2}\right)=1 \text {. }
\end{aligned}
$$

In those cases where the range of the summation indices has not been given explicitly, we mention that $1 \leq i_{1} \leq m, 1 \leq j_{1} \leq r_{i_{1}}$, and there are similar bounds for $\left(i_{2}, j_{2}\right),\left(i^{\prime}, j^{\prime}\right)$ and $\left(i^{\prime \prime}, j^{\prime \prime}\right)$. In order to solve these equations, we introduce a constant $c$ where

$$
c=\sum_{i^{\prime}, j^{\prime}} x\left(1, i^{\prime}, j^{\prime}\right) f_{i^{\prime}, \geq j^{\prime}}+\sum_{i^{\prime}, i^{\prime \prime}, j^{\prime}, j^{\prime \prime}} x\left(2, i^{\prime}, i^{\prime \prime}, j^{\prime}, j^{\prime \prime}\right) F_{i^{\prime}, i^{\prime \prime}, \geq j^{\prime}, \geq j^{\prime \prime}}
$$

and (3) can then be rewritten as follows:

$$
x(1, i, j)=c b_{i j}
$$

Therefore, the system (4) can be solved autonomously as follows:

$$
x\left(2, i_{1}, i_{2}, j_{1}, j_{2}\right)=c z\left(2, i_{1}, i_{2}, j_{1}, j_{2}\right)
$$

where $z(\cdot)$ is the solution of $(4)$ when the function $x(1, i, j)$ is replaced by $b_{i j}$. The constant $c$ can be obtained from the equation

$$
c^{-1}=\sum_{i, j} b_{i j}+\sum_{i_{1}, i_{2}, j_{1}, j_{2}} z\left(2, i_{1}, i_{2}, j_{1}, j_{2}\right)
$$

and the loss probability $p_{\text {loss }}$ can then be computed as follows: 


$$
p_{\text {loss }}=\sum_{i_{1}, i_{2}, j_{1}, j_{2}} x\left(2, i_{1}, i_{2}, j_{1}, j_{2}\right)\left(1-f_{i_{1}, \geq j_{1}}-f_{i_{2}, \geq j_{2}}+F_{i_{1}, i_{2}, \geq j_{1}, \geq j_{2}}\right) .
$$
How many variables are involved? The system (4) contains $\left(\sum_{i=1}^{m} r_{i}\right)^{2}$ variables $z\left(2, i_{1}, i_{2}, j_{1}, j_{2}\right)$
but the symmetry condition

$$
z\left(2, i_{2}, i_{1}, j_{2}, j_{1}\right)=z\left(2, i_{1}, i_{2}, j_{1}, j_{2}\right)
$$

allows us to keep only those variables for which $i_{1}<i_{2}$ or $i_{1}=i_{2}$ and $j_{1}<j_{2}$. An elementary combinatorics gives

$$
N=\frac{1}{2}\left(\left(\sum_{i=1}^{m} r_{i}\right)^{2}+\sum_{i=1}^{m} r_{i}\right)
$$

for the number of variables kept. Note that $\sum_{i=1}^{m} r_{i}$ is the number of terms in the expansion (1). (A term with $b_{i j}=0$ must be counted if $b_{i j^{\prime}} \neq 0$ for some $j^{\prime}>j$ ). Thus the dimensionality of the problem is of quadratic order, and so standard (non-improved) computer algorithms will require an order $\left(\sum r_{i}\right)^{6}$ of elementary operations.

However, a more attentive analysis allows us to reduce the order by using an appropriate iterative procedure. Let us consider an absorbing Markov chain $\left(\nu_{n}, i_{n}, j_{n}\right)$ starting with the state $\left(\nu_{0}, i_{0}, j_{0}\right)$, where $\nu_{0}=1$ and $p\left(i_{0}=i, j_{0}=j\right)=b_{i j}$. As to the subsequent values of $\left(\nu_{n}, i_{n}, j_{n}\right)$, the transitions coincide with those of the original Markov chain without absorptions, except that each transition to a state with $\nu_{n}=1$ now results in absorption. We can therefore retain our previous notation and we have the following iterative scheme for the distributions $x_{n}\left(i_{1}, i_{2}, j_{1}, j_{2}\right)$ of the absorbing Markov chain at the stage $n \geq 1$ :

$$
\begin{gathered}
x_{1}\left(i_{1}, i_{2}, j_{1}, j_{2}\right)=\frac{1}{2} \sum_{k=0}^{r_{i_{1}}-j_{1}} f_{i_{1} k b_{i_{2} j_{2}} b_{i_{1}, j_{1}+k}+\frac{1}{2} \sum_{k=0}^{r_{i_{2}}-j_{2}} f_{i_{2} k^{k} b_{i_{1} j_{1}} b_{i_{2}, j_{2}}+k}}^{x_{n}\left(i_{1}, i_{2}, j_{1}, j_{2}\right)=} \sum_{k=0}^{r_{i_{1}}-j_{1}} \sum_{l=0}^{r_{i_{2}}-j_{2}} x_{n-1}\left(i_{1}, i_{2}, j_{1}+k, j_{2}+\ell\right) F_{i_{1}, i_{2}, k, l} \\
+\sum_{k=0}^{r_{i_{1}}-j_{1}} \sum_{i^{\prime}} \sum_{l=0}^{r_{i^{\prime}}} x_{n-1}\left(i_{1}, i^{\prime}, j_{1}+k, l\right) b_{i_{2} j_{2}} F_{i_{1}, i^{\prime}, k, \geq l} \\
+\sum_{k=0}^{r_{i_{2}}-j_{2}} \sum_{i^{\prime}} \sum_{l=0}^{r_{i^{\prime}}} x_{n-1}\left(i^{\prime}, i_{2}, l, j_{2}+k\right) b_{i_{1} j_{1}} F_{i^{\prime}, i_{2}, \geq l, k} \text { for } n \geq 2 .
\end{gathered}
$$

It can easily be seen by a standard regenerative argument that

where

$$
p_{\text {loss }}=\frac{\sum_{n=2}^{\infty} Y_{n}}{1+\sum_{n=1}^{\infty} X_{n}}
$$

$$
X_{n}=\sum_{i_{1}, i_{2}, j_{1}, j_{2}} x_{n}\left(i_{1}, i_{2}, j_{1}, j_{2}\right)
$$




$$
Y_{n}=\sum_{i_{1}, i_{2}, j_{1}, j_{2}} x_{n-1}\left(i_{1}, i_{2}, j_{1}, j_{2}\right)\left(1-f_{i_{1}}, \geq j_{1}-f_{i_{2}, \geq j_{2}}+F_{i_{1}, i_{2}, \geq j_{1} \geq j_{2}}\right) .
$$

For many widely used distributions, both the series in (15) converge sufficiently rapidly. For example, if the service-time distribution has an increasing failure rate, then $X_{n}$ and $Y_{n}$ tend to zero in an exponential manner. In general, one cannot assure good estimates for $X_{n}$ and $Y_{n}$, but both certainly vanish as $n$ goes to infinity. Thus, in either case, one needs a finite number $N$ of iterations for a given admissible error in the value of $p_{\text {loss }}$. How many elementary operations are needed? It can be seen that, at each iteration, an $\left(i_{1}, i_{2}\right)$ variable at step $n$ is connected to $\left(i^{\prime}, i_{2}\right)$ and $\left(i_{1}, i^{\prime}\right)$ variables at step $n-1$. It therefore suffices to take the number $L$ of elementary operations each consisting of a multiplication and an addition, required for each iteration, to be given by

$$
L=\sum_{i, j} r_{i} r_{j}\left(\sum_{i^{\prime}} r_{i^{\prime}} r_{j}+\sum_{j^{\prime}} r_{i} r_{j^{\prime}}\right)=2\left(\sum_{i=1}^{m} r_{i}\right)^{2} \sum_{i=1}^{m} r_{i}^{2}
$$

The maximal value of $L$ is

$$
L_{\text {max }}=2\left(\sum_{i=1}^{m} r_{i}\right)^{4}
$$

whereas if $r_{i} \leq R, 1 \leq i \leq m$, then

$$
L \leq 2 R\left(\sum_{i=1}^{m} r_{i}\right)^{3}
$$

In practical computations, very large values of $\sum r_{i}$ can be expected to occur infrequently, otherwise the model of $b(x)$ would have a low chance of being assured statistically, so that an estimate of $2 N L$ would seem to be quite satisfactory. Of course, it remains to be seen how large $N$, the required number of iterations, will be in practical applications.

Special Cases: We consider two special cases: (1) by setting $r_{i}=1(i=1, \ldots, m)$ we obtain a "mixture" of exponentials; (2) by setting $m=1$ we obtain a "Hyper-Erlangian" model.

Mixture of Exponentials: In this case, $b(x)$ takes the form

$$
b(x)=\sum_{i=1}^{m} b_{i} \lambda_{i} \exp \left(-\lambda_{i} x\right), \quad x>0 .
$$

We can simplify the notation as follows:

$$
\begin{gathered}
x_{n}\left(i_{1}, i_{2}, 1,1\right)=x_{n}\left(i_{1}, i_{2}\right) \\
b_{i 1}=b_{i}
\end{gathered}
$$

and we introduce the following constants;

$$
\begin{aligned}
& \alpha_{i}^{+}=\int_{0}^{\infty}\left(1-\exp \left(-\lambda_{i} x\right)\right) d A(x), \alpha_{i}^{-}=1-\alpha_{i}^{+} \\
& \alpha_{i j}^{+}=\int_{0}^{\infty}\left(1-\exp \left(-\lambda_{i} x\right)\right)\left(1-\exp \left(-\lambda_{j} x\right)\right) d A(x) \\
& \alpha_{\overline{i j}^{+}}^{+}=\int_{0}^{\infty} \exp \left(-\lambda_{i} x\right)\left(1-\exp \left(\lambda_{j} x\right)\right) d A(x)
\end{aligned}
$$




$$
\begin{aligned}
& \alpha_{i j}^{+}=\int_{0}^{\infty}\left(1-\exp \left(-\lambda_{i} x\right)\right) \exp \left(-\lambda_{j} x\right) d A(x) \\
& \alpha_{i j}^{-}=\int_{0}^{\infty} \exp \left(-\lambda_{i} x-\lambda_{j} x\right) d A(x) .
\end{aligned}
$$

Equations (13) and (14) can now be written as follows:

$$
\begin{gathered}
x_{1}\left(i_{1}, i_{2}\right)=\frac{1}{2} b_{i_{1}} b_{i_{2}}\left(\alpha_{i_{1}}^{-}+\alpha_{i_{2}}^{-}\right) \\
x_{n}\left(i_{1}, i_{2}\right)=x_{n-1}\left(i_{1}, i_{2}\right) \alpha_{i_{1} i_{2}}^{-}+\sum_{i^{\prime}}^{-} x_{n-1}\left(i^{\prime}, i_{2}\right) \alpha_{i^{\prime} i_{2}}^{+} b_{i_{1}} \\
+\sum_{i^{\prime}} x_{n-1}\left(i_{1}, i^{\prime}\right) \alpha_{i_{1} i^{\prime}}^{-} b_{i_{2}}, \quad n \geq 2 .
\end{gathered}
$$

Each iteration requires $L=2 \mathrm{~m}^{3}$ elementary operations, not counting the symmetry reduction.

"Hyper-Erlangian" Model: In this case $b(x)$ takes the following form:

$$
b(x)=\sum_{j=1}^{r} b_{j} \frac{\lambda^{j} x^{j-1}}{(j-1) !} \exp (-\lambda x), \quad x>0 .
$$

We use the following simplified notation:

$$
\begin{aligned}
& \lambda_{1}=\lambda, r_{1}=r, \\
& x_{n}\left(1,1, j_{1}, j_{2}\right)=x_{n}\left(j_{1}, j_{2}\right) \text {, } \\
& f_{1 j}=f_{j}=\int_{0}^{\infty} \frac{(\lambda x)^{j}}{j !} \exp (-\lambda x) d A(x) \\
& f_{1, \geq j}=f_{\geq j}=1-\sum_{j^{\prime}=0}^{j-1} f_{j^{\prime}} \\
& F_{1,1, j_{1}, j_{2}}=F_{j_{1} j_{2}}=\frac{\lambda^{j_{1}+j_{2}}}{j_{1} ! j_{2} !} \int_{0}^{\infty} x^{j_{1}+j_{2}} \exp (-2 \lambda x) d A(x) \\
& F_{\geq j_{1}, j_{2}}=f_{j_{2}}-\sum_{j^{\prime}=0}^{j_{1}-1} F_{j^{\prime}, j_{2}} \\
& F_{j_{1}, \geq j_{2}}=f_{j_{1}}-\sum_{j^{\prime}=0}^{j_{2}-1} F_{j_{1}, j^{\prime}} \\
& F_{\geq j_{1}, \geq j_{2}}=f_{\geq j_{1}}-\sum_{j^{\prime}=0}^{j_{2}-1} F_{\geq j_{1}, j^{\prime}}
\end{aligned}
$$


Equations (13) and (14) then become:

$$
\begin{gathered}
x_{1}\left(j_{1}, j_{2}\right)=\frac{1}{2} \sum_{k=0}^{r-j_{1}} f_{k} b_{j_{2}} b_{j_{1}+k}+\frac{1}{2} \sum_{k=0}^{r-j_{2}} f_{k} b_{j_{1}} b_{j_{2}+k} \\
x_{n}\left(j_{1}, j_{2}\right)=\sum_{k=0}^{r-j_{1}} \sum_{l=0}^{r-j_{2}} F_{k, l} x_{n-1}\left(j_{1}+k, j_{2}+l\right) \\
+\sum_{k=0}^{r-j_{1}} \sum_{l=0}^{r} F_{k, \geq l} b_{j_{2}} x_{n-1}\left(j_{1}+k, l\right) \\
+\sum_{k=0}^{r-j_{2}} \sum_{l=0}^{r} F \geq l, k^{b} j_{1} x_{n-1}\left(l, j_{2}+k\right) \quad \text { for } n \geq 2 .
\end{gathered}
$$

Each iteration requires $L=2 r^{3}$ elementary operations, not counting the symmetry reduction.

\section{The GI/G/2/0 Queue in Discrete Time}

In this section we summarize some results given in [3] for the discrete-time GI/G/2/0 queue and we indicate how they can be used to approximate continuous-time systems. Introducing a time unit $\Delta$ of arbitrary duration, we define the distributions of $X$, the interarrival time, and $Y$, the service time, as follows:

$$
\begin{array}{ll}
P(X=k \Delta)=p_{k} & k=1,2, \ldots \\
P(Y=k \Delta)=q_{k} & k=1,2, \ldots
\end{array}
$$

The system state is defined by the triplet $(i, j, k)$ where $i \quad(i=1,2, \ldots)$ is the number of time units remaining until the next customer arrives, $j(j=0,1, \ldots)$ is the number of time units remaining until server 1 completes its current service $(j=0$ implies that server 1 is idle), while $k$ $(k=0,1, \ldots)$ refers in a similar way to server $2 . P(i, j, k)$ is the steady-state probability that the system is in state $(i, j, k)$. We also define $\phi_{j}=P(1, j+1,0)+P(1, j+1,1)$ for $j \geq 1$,

$$
\alpha=\frac{1}{2}[P(1,1,1)+P(1,1,0)+P(1,0,1)+P(1,0,0)],
$$

and the renewal function

$$
\begin{gathered}
\gamma_{0}=1 \\
\gamma_{t}=\sum_{v=1}^{t} p_{v} \gamma_{t-v}(t>0)
\end{gathered}
$$

with the associated function $A(n, t)$ for $t \geq 1$, where

$$
\begin{gathered}
A(1, t)=p_{t} \\
A(n, t)=A(n-1, t+1)+p_{t} \gamma_{n-1} \quad(n>1) .
\end{gathered}
$$

It is shown in [3] that the $\phi_{k}(k \geq 1)$ satisfy the following system of equations: 


$$
\phi_{k}=\sum_{t=1}^{\infty}\left[\sum_{n=1}^{\infty}\left(q_{n} \phi_{k+t+n-1}+q_{k+t+n-1} \phi_{n}\right) A(n, t)+p_{t} q_{k+t} \alpha\right]
$$

When $X$ and $Y$ are bounded, the above system can be solved numerically to obtain $\phi_{k}$ values in units of $\alpha$, from which $p_{\text {loss }}$ can be obtained as follows:

$$
p_{\text {loss }}=\frac{\sum_{t=1}^{\infty} Q(t) \Phi(t) \gamma_{t}}{\sum_{t=1}^{\infty} Q(t) \Phi(t) \gamma_{t}+\Phi(0)+\alpha},
$$

where $Q(t)$ is the complementary cumulative distribution function of the service time, thus:

$$
Q(t)=\sum_{j=t+1}^{\infty} q_{j} \quad t=0,1, \ldots
$$

and

$$
\Phi(t)=\sum_{j=t+1}^{\infty} \phi_{j} \quad t=0,1, \ldots
$$

In order to approximate general continuous distribution functions $A(x)$ and $B(x)$ for the interarrival time and service time respectively, one approach is to truncate both distributions at a point $x=L$, such that $\operatorname{Min}\{A(L), B(L)\}=1-\epsilon$ where $\epsilon$ is a chosen probability close to 0 . We then obtain the truncated distributions $A^{*}(x)=A(x) / A(L)$ and $B^{*}(x)=B(x) / B(L)$, both of which are defined on the domain $0 \leq x \leq L$.

We introduce a time unit $\Delta$ such that $m \Delta=L$ where $m$ is a sufficiently large integer, and then make use of the discrete distributions given by

$$
\left.\begin{array}{l}
p_{i}=A^{*}(i \Delta)-A^{*}(i \Delta-\Delta) \\
q_{i}=B^{*}(i \Delta)-B^{*}(i \Delta-\Delta)
\end{array}\right\} \quad i=1,2, \ldots, m .
$$

Calculation of approximate $p_{\text {loss }}$ values is carried out using equations (27) and (28). The quality of the approximations clearly depends upon the values chosen for $\epsilon$ and $m$.

\section{Numerical Examples}

In this section we describe some preliminary numerical investigations which allow us to illustrate, and make some comparisons between, the matrix-exponential approach and the discrete-time approach to the GI/G/2/0 queue. The examples involve the following family of distributions $B_{n} \quad(n=1,2, \ldots)$ having the density:

$$
b_{n}(x)=c_{n}(1-\cos (2 \pi x)) \exp (-x), \quad 0 \leq x \leq n
$$

where $c_{n}=\left(1+\frac{1}{4 \pi^{2}}\right)(1-\exp (-n))^{-1}$.

For convenience, as $n$ goes to infinity we replace $B_{n}, b_{n}$ and $c_{n}$ by $B, b$ and $c$ respectively. The densities corresponding to finite $n$ are truncated versions of $b(x)$. The latter, which is shown in Figure 1, is given by Asmussen and Bladt [1] as an example of a density function that does not belong to the class of phase-type distributions but does belong to the larger class of matrixexponential distributions. The matrix-exponential representation is: 


$$
\begin{aligned}
& b(x)=\underline{a} \exp (\underline{T} x) \underline{s} \text { where } \\
& \qquad \underline{a}=(1,1,1), \underline{T}=\left(\begin{array}{ccc}
2 \pi i-1 & 0 & 0 \\
0 & -2 \pi i-1 & 0 \\
0 & 0 & -1
\end{array}\right), \underline{s}=\left(\begin{array}{c}
-c / 2 \\
-c / 2 \\
c
\end{array}\right),
\end{aligned}
$$

and, in the form of (21), $b(x)$ is a mixture of exponentials in which $m=3, \lambda_{1}=1-2 \pi i$, $\lambda_{2}=1+2 \pi i, \lambda_{3}=1$ and $b_{1}=-c /\left(2 \lambda_{1}\right), b_{2}=-c /\left(2 \lambda_{2}\right), b_{3}=c / \lambda_{3}$.

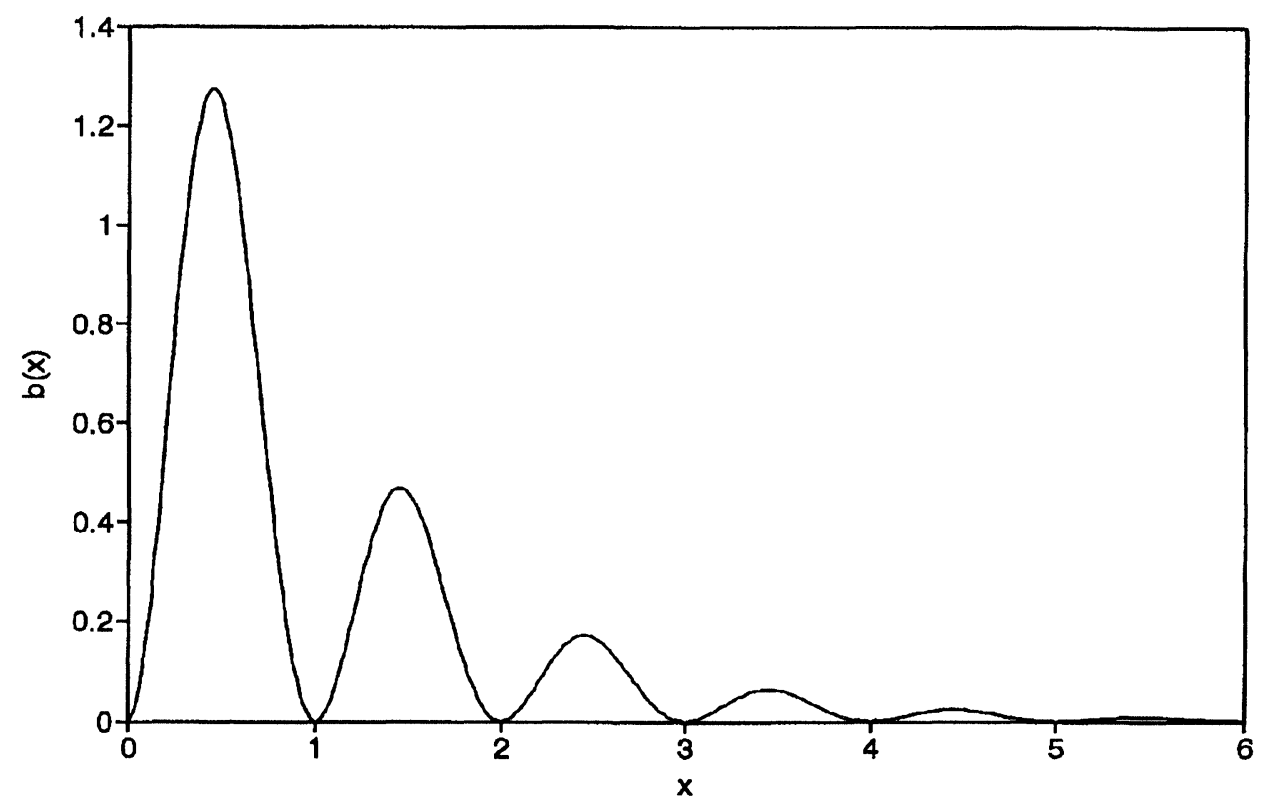

Figure 1: The density $b(x)$

Using a Fortran program based upon equations (15)-(17) and (21)-(23), the queues $\mathrm{M} / \mathrm{B} / 2 / 0$, $\mathrm{B}_{n} / \mathrm{B} / 2 / 0$ and $\mathrm{B}_{n} / \mathrm{B}_{n} / 2 / 0$ have been studied. The first of these queues has $p_{\text {loss }}$ values given by the well-known Erlang Loss formula; this queue is of interest here partly to validate the computational method and also to study the rate at which the algorithm converges. Obviously, one expects the rate of convergence to depend upon the traffic intensity $s / a$, and this can be seen in Figure 2 where the estimate $p_{\text {loss }}^{(N)}$ of the steady-state loss probability is plotted against the iteration number $N$; i.e., modifying (15),

$$
p_{\text {loss }}^{(N)}=\frac{\sum_{n=2}^{N} Y_{n}}{1+\sum_{n=1}^{N} X_{n}} .
$$




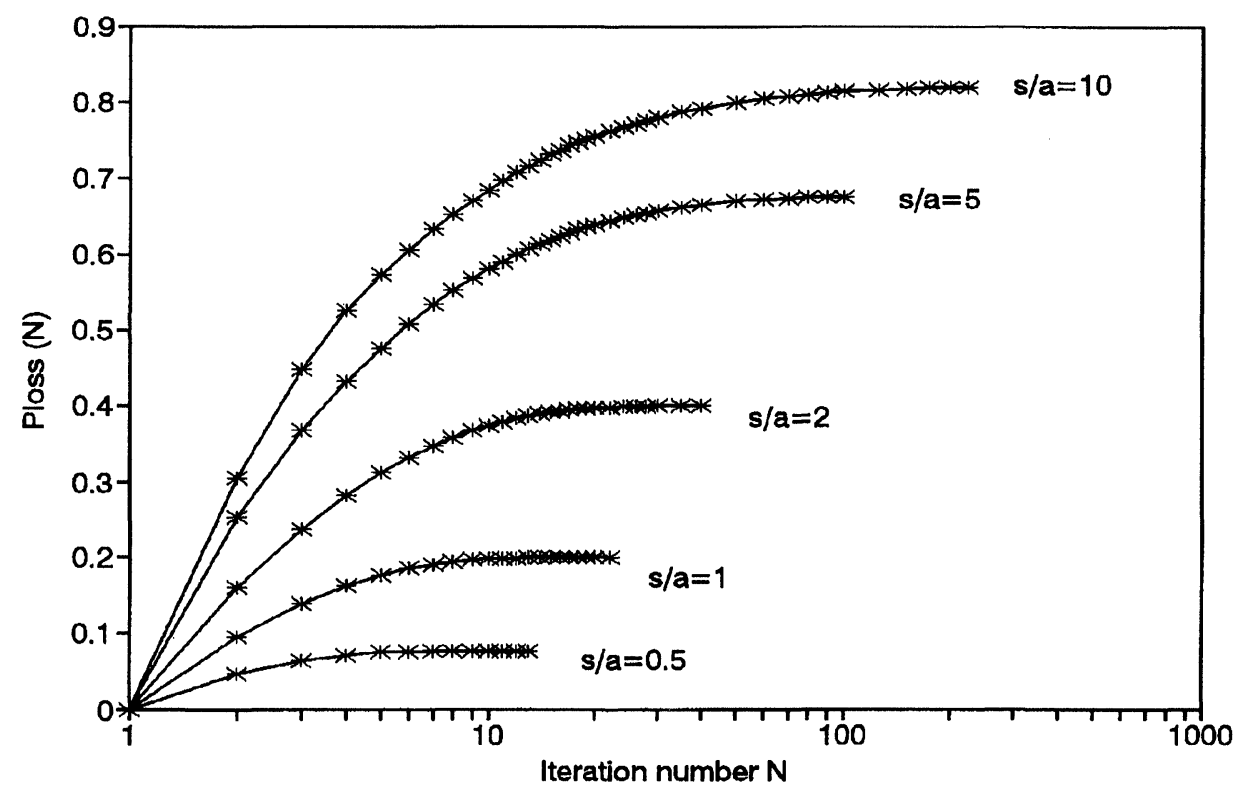

Figure 2: Estimates of the loss probability for the queue $\mathrm{M} / \mathrm{B} / 2 / 0$ with varying traffic intensity $(s / a)$

In all the studies described here, the iterative process was terminated when the loss probability had been calculated to an accuracy of 0.00001 ; i.e., after $N^{*}$ iterations when $p_{\text {loss }}^{\left(N^{*}\right)}-p_{\text {loss }}^{\left(N^{*}-1\right)}<$ 0.00001. It was found that the number $N^{*}$ was almost exactly proportional to the traffic intensity; see Figure 3. 


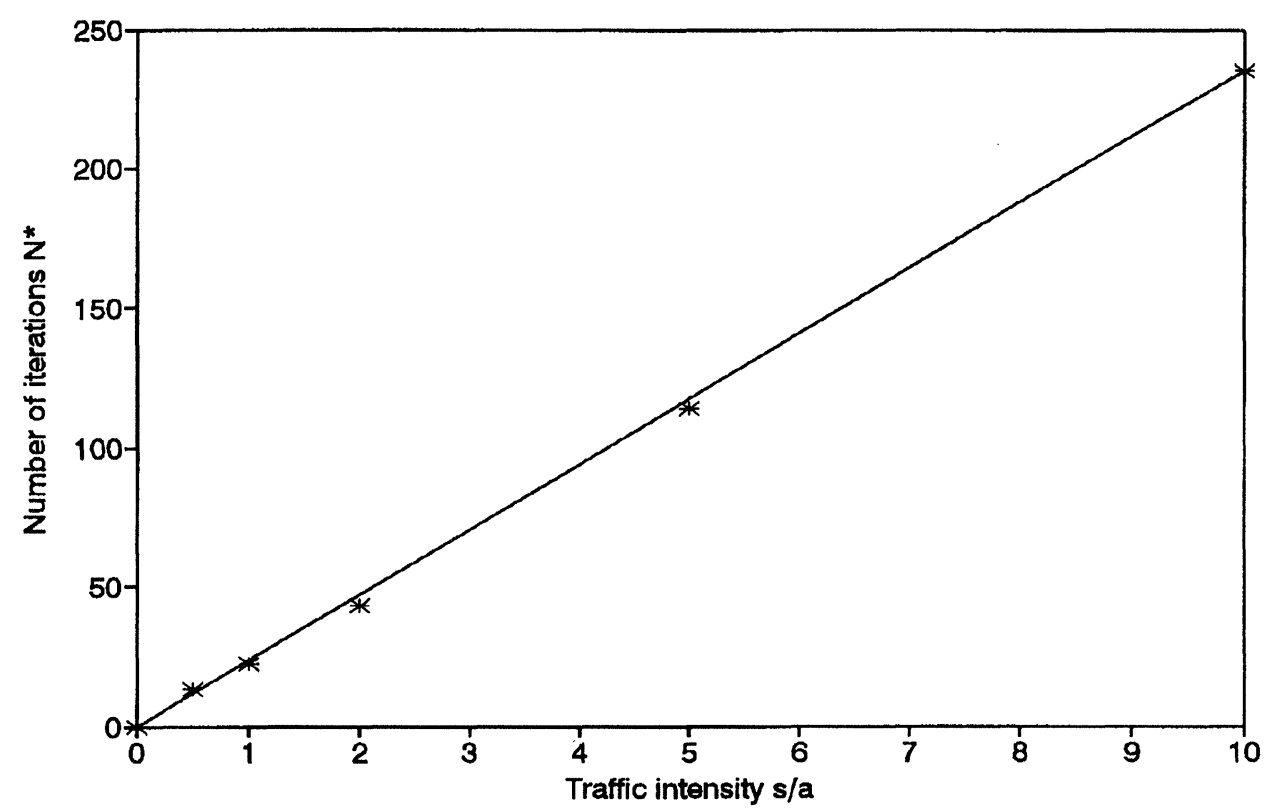

Figure 3: Variation of the rate of convergence $\left(N^{*}\right)$ with the traffic intensity $(s / a)$ for the queue $\mathrm{M} / \mathrm{B} / 2 / 0$

Next we consider the queue $\mathrm{B}_{n} / \mathrm{B} / 2 / 0$ for a range of $n$ values, in each case calculating loss probabilities by both the matrix-exponential model and the discrete-time model. In the latter case, the distribution $B_{14}$ (i.e. corresponding to $L=14$ ) was taken to be a sufficiently accurate representation of $B$, the truncated tail probability having a value $\epsilon$ that is less than $10^{-6}$. The number of time points $m$ used in the approximation took values up to a maximum of 7,000 , and the algorithm was terminated when the value of $p_{\text {loss }}$ calculated for the approximating problem had been determined to an accuracy of 0.0001 . The results for the two different approaches, using programs running on a Vax 11/780 mainframe computer, are compared in Table 1 . For the $\mathrm{B}_{n} / \mathrm{B}_{n} / 2 / 0$ queue, in which both the interarrival time and the service-time distributions have the form $B_{n}$ with $n$ finite, the matrix-exponential method is no longer applicable as an exact method. In this case, only the discrete-time approach has been used and the results are given in Table 2.

The results in Table 1 show good agreement between the two methods. The $p_{\text {loss }}$ values for the matrix-exponential method are, of course, exact to the prescribed accuracy, while those for the discrete-time method are approximate but with errors that are quite low. For the examples using 7,000 time points, the errors are about $0.3 \%$, which is comparable with results for other systems described in [3]. In general, the discrete-time method was heavy in its use of computing time (see, for example, the c.p.u. times listed in Table 2) while, for the matrix-exponential method, in each case the c.p.u. time was negligible. It is interesting to note that in the latter case the number $N^{*}$ of iterations could be predicted quite closely using the graph in Figure 3 for the $\mathrm{M} / \mathrm{B} / 2 / 0$ queue. 
Table 1: Results for the queue $\mathrm{B}_{n} / \mathrm{B} / 2 / 0$

\begin{tabular}{|c|c|c|c|c|c|c|c|}
\hline \multicolumn{4}{|c|}{ Matrix Exponential Method } & \multicolumn{4}{|c|}{ Discrete-Time Method } \\
\hline$n$ & $s / a$ & $p_{\text {loss }}$ & $N^{*}$ & $m$ & $s / a$ & $p_{\text {loss }}$ & $\%$ error \\
\hline \multirow[t]{3}{*}{1} & 2.2451 & 0.3512 & 50 & 1,400 & 2.2319 & 0.3465 & 1.34 \\
\hline & & & & 2,800 & 2.2384 & 0.3489 & 0.65 \\
\hline & & & & 7,000 & 2.2424 & 0.3503 & 0.26 \\
\hline \multirow[t]{3}{*}{2} & 1.4251 & 0.2227 & 29 & 1,400 & 1.4222 & 0.2195 & 1.44 \\
\hline & & & & 2,800 & 1.4237 & 0.2211 & 0.72 \\
\hline & & & & 7,000 & 1.4245 & 0.2220 & 0.31 \\
\hline \multirow[t]{3}{*}{5} & 1.0334 & 0.1689 & 22 & 1,400 & 1.0332 & 0.1665 & 1.42 \\
\hline & & & & 2,800 & 1.0333 & 0.1677 & 0.71 \\
\hline & & & & 7,000 & 1.0334 & 0.1684 & 0.30 \\
\hline \multirow[t]{3}{*}{10} & 1.0004 & 0.1664 & 22 & 1,400 & 1.0004 & 0.1640 & 1.44 \\
\hline & & & & 2,800 & 1.0004 & 0.1652 & 0.72 \\
\hline & & & & 7,000 & 1.0004 & 0.1660 & 0.24 \\
\hline \multirow[t]{3}{*}{14} & 1.0000 & 0.1664 & 22 & 1,400 & 1.0000 & 0.1640 & 1.44 \\
\hline & & & & 2,800 & 1.0000 & 0.1652 & 0.72 \\
\hline & & & & 7,000 & 1.0000 & 0.1659 & 0.30 \\
\hline
\end{tabular}

Table 2: Results for the queue $\mathrm{B}_{n} / \mathrm{B}_{n} / 2 / 0$ using the Discrete-Time Method

\begin{tabular}{|c|c|c|c|}
\hline$n$ & $m$ & $p_{\text {loss }}$ & $\begin{array}{c}\text { c.p.u. } \\
\text { min:sec }\end{array}$ \\
\hline \multirow{2}{*}{1} & 500 & 0.0460 & $0: 03.4$ \\
& 1,000 & 0.0463 & $0: 15.5$ \\
& 2,000 & 0.0464 & $1: 08.4$ \\
& 4,000 & 0.0465 & $4: 55.8$ \\
\hline & & & \\
2 & 1,000 & 0.1143 & $0: 26.3$ \\
& 2,000 & 0.1145 & $1: 52.2$ \\
& 4,000 & 0.1147 & $8: 20.6$ \\
\hline \multirow{3}{*}{5} & 1,000 & 0.1591 & $0: 18.7$ \\
& 2,500 & 0.1598 & $2: 13.4$ \\
& 5,000 & 0.1601 & $9: 48.1$ \\
\hline \multirow{2}{*}{10} & 1,000 & 0.1639 & $0: 26.9$ \\
& 2,000 & 0.1651 & $1: 57.2$ \\
& 5,000 & 0.1659 & $13: 07.8$ \\
\hline
\end{tabular}


Of course, the distribution $B$ is defined precisely and is of low dimension $\left(\Sigma r_{i}=3\right)$. In a more general case, it might be necessary to fit an appropriate ME density to represent an actual service-time distribution, for example by using the approach described in [2]. In this case, the speed of the matrix-exponential method would clearly depend upon the dimension of the ME representation used, and the accuracy would depend upon how well the actual service-time distribution was represented by the fitted ME density. In the absence of an appropriate ME representation, the discrete-time method is sufficiently flexible to give acceptable approximations, as shown in Table 2 for the $\mathrm{B}_{n} / \mathrm{B}_{n} / 2 / 0$ queue.

Finally, it should be noted that the form of the discrete-time distribution fitted to the $B_{n}$ densities in this paper, is not necessarily the most appropriate one for all applications. In the trade-off between computing time and accuracy, simpler discrete-time approximations could be devised by combining fewer time points, and hence shorter computing times, with a less precise fit to the distribution's shape, for example by matching moments. The implications of this trade-off will be the subject of further research.

\section{Concluding Remarks}

In this paper we have presented a computationally efficient method for calculating steadystate loss probabilities in the queue GI/G/2/0 when the service-time distribution can be represented by a matrix-exponential distribution. We have also considered an alternative discrete-time approximation method. Preliminary numerical studies suggest that the two approaches are complementary in that the preferred choice in any application will depend largely upon the nature of the service-time distribution and how accurately it can be modelled by each of the two approaches.

\section{References}

[1] Asmussen, S. and Bladt, M., Renewal theory and queueing algorithms for matrixexponential distributions, Pre-print (1993).

[2] Asmussen, S. and Nerman, O., Fitting phase-type distributions via the EM algorithm, In: Symposium i Anvendt Statistik (ed. by K. Vest Nielsen) (1991), 335-346.

[3] Atkinson, J.B., The general two-server queueing loss system: discrete-time analysis and numerical approximation of continuous-time systems, submitted to J. Opl. Res. Soc. (1993).

[4] Cox, D.R., A use of complex probabilities in the theory of stochastic processes, Proc. Camb. Philos. Soc. 51 (1953), 313-319.

[5] Neuts, M.F., Matrix-Geometric Solutions in Stochastic Models - An Algorithmic Approach, John Hopkins University Press, Baltimore 1981. 


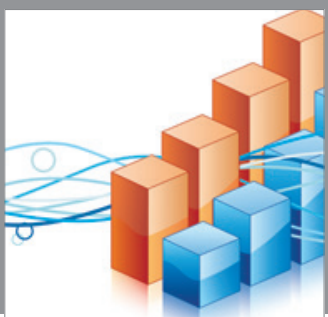

Advances in

Operations Research

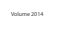

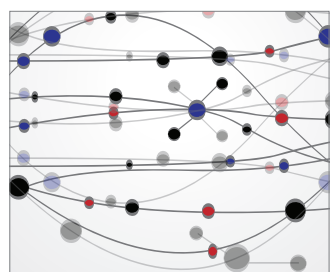

\section{The Scientific} World Journal
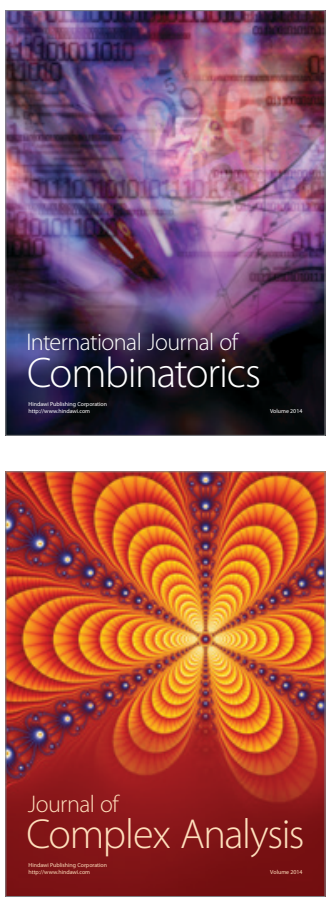

International Journal of

Mathematics and

Mathematical

Sciences
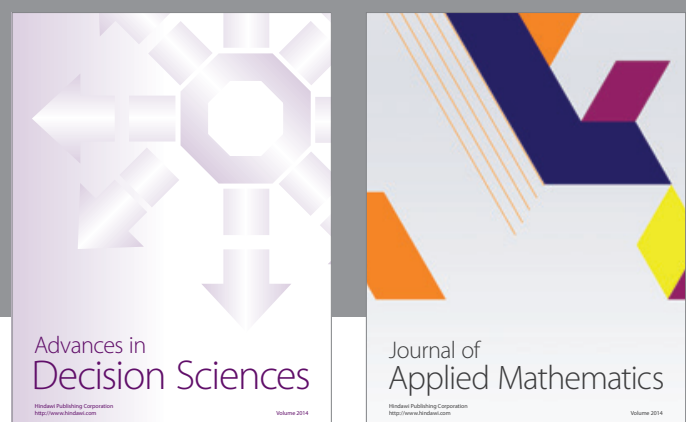

Journal of

Applied Mathematics
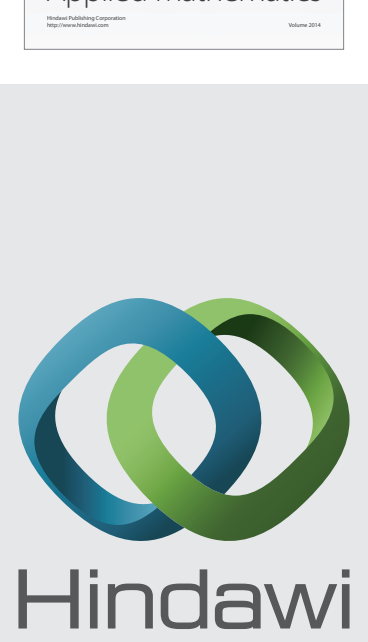

Submit your manuscripts at http://www.hindawi.com
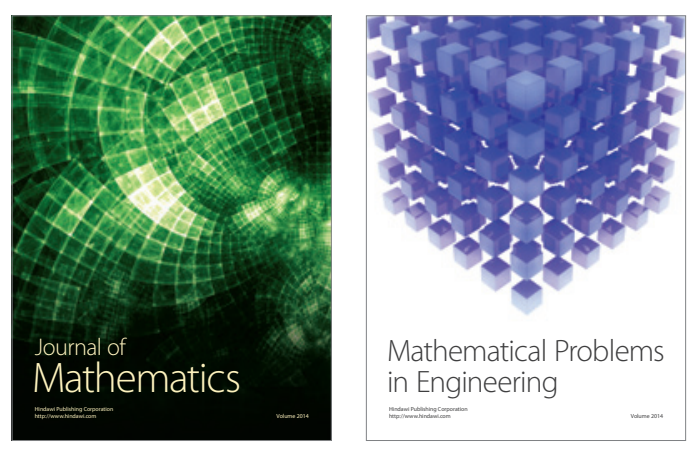

Mathematical Problems in Engineering
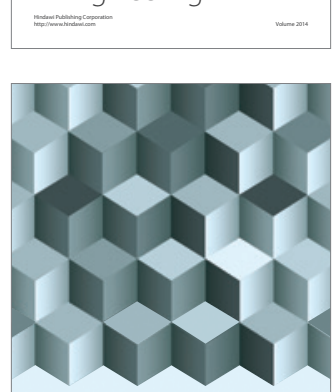

Journal of

Function Spaces
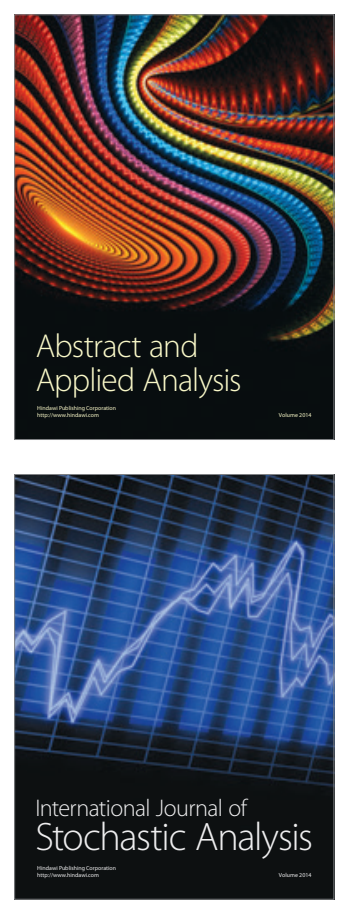

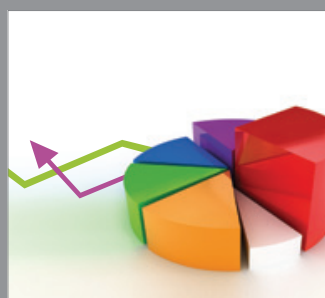

ournal of

Probability and Statistics

Promensencen
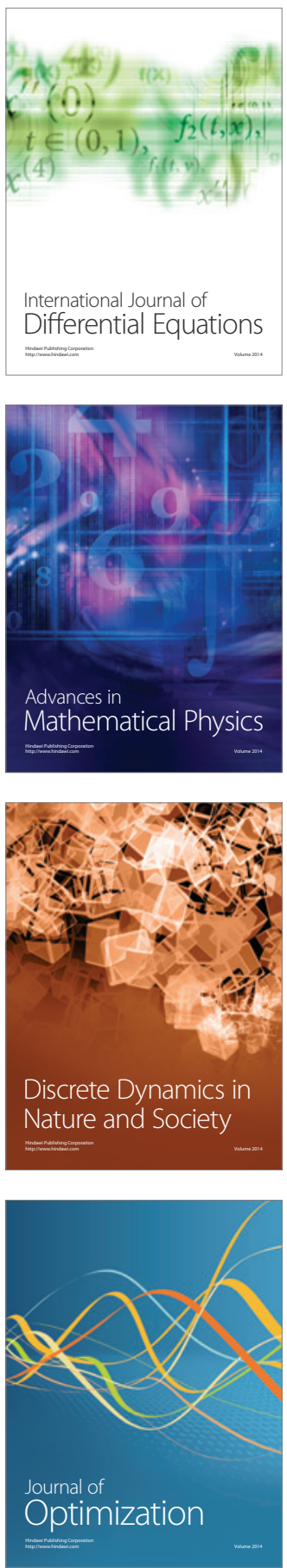